\title{
Effect of Grape Seed Proanthocyanidins on Tumor Vasculogenic Mimicry in Human Triple-negative Breast Cancer Cells
}

\author{
Yun-Yan Luan', Zi-Min Liu', Jin-Yi Zhong², Ru-Yong Yao³, Hong-Sheng Yu ${ }^{1 *}$
}

\begin{abstract}
Vasculogenic mimicry (VM) refers to the unique ability of highly aggressive tumor cells to mimic the pattern of embryonic vasculogenesis, which was associated with invasion and metastasis. The grape seed proanthocyanidins (GSPs) had attracted much attention as a potential bioactive anti-carcinogenic agent. However, GSPs regulation of VM and its possible mechanisms in a triple-negative breast cancer cells (TNBCs) remain not clear. Therefore, we examined the effect of GSPs on VM information in HCC1937 cell model. In this study, we identified the VM structure via the three-dimensional (3D) matrix in vitro. Cell viability was measured using the CCK8 assay. The effects of GSPs on human triple-negative breast cancer cells (TNBCs) HCC1937 in terms of related proteins of VM information were determined using western blot analysis. In vitro, the tubular networks were found in highly invasive $\mathrm{HCC} 1937$ cells but not in the non-invasive MCF-7 cells when plated on matrigel. The number of vascular channels was significantly reduced when cells were exposed in GSPs $(100 \mu \mathrm{g} / \mathrm{ml})$ and GSPs $(200 \mu \mathrm{g} / \mathrm{ml})$ groups (all $p<0.001$ ). Furthermore, we found that treatment with GSPs promoted transition of the mesenchymal state to the epithelial state in $\mathrm{HCC} 1937$ cells as well as reducing the expression of Twist1 protein, a master EMT regulator.GSPs has the ability to inhibit VM information by the suppression of Twist1 protein that could be related to the reversal of epithelial-to-mesenchymal (EMT) process. It is firstly concluded that GSPs may be an potential anti-VM botanical agent for human TNBCs.
\end{abstract}

Keywords: Human triple-negative breast cancer cells - vasculogenic mimicry - grape seed proanthocyanidins

Asian Pac J Cancer Prev, 16 (2), 531-535

\section{Introduction}

Epidemiological studies have shown that breast cancer is the leading cause of cancer-associated deaths in women on a global scale. The vast majority of cancer death cases are due to distant metastases (Redig et al., 2013). Especially triple-negative breast cancer (TNBC) subtype, accounting for approximately $10-15 \%$ of all breast cancers, displays highly aggressive, propensity to relapse and metastasis and dismal prognosis (Taylor et al., 2013; Han et al., 2014). Clinically, the malignant and heterogeneous subtype, which was lack of the expression of Estrogen receptor (ER), Progesterone receptor (PR) and Human Epidermal Growth Factor receptor (HER2), was referred to as TNBC (Di Cosimo et al., 2010; Taylor et al., 2013; Rhodes et al., 2014). To date, there lacks specific therapeutic targets for TNBC except for chemotherapy. Consequently, it is critical need to explore a novel perspective therapeutics for TNBC, as most of patients experience rapid recurrence and distant metastasis.

To the best of our knowledge, malignant tumors growth, invasion, and metastasis are dependent on neovascularization. Previously, we focused most attention on endothelial cell-lined vessels. However, the discovery of vasculogenic mimicry (VM) may challenge the assumption that endothelial cell-lined vessels are the only pathways supplemented with nourishment (Maniotis et al., 1999; Seftor et al., 2012). Cells of highly aggressive tumor are able to form structured vascular channels surrounded by the tumor cells without participation of endothelial cells (ECs) and independent of angiogenesis, which were so-called vasculogenic mimicry (VM) (Chen et al., 2012; Seftor et al., 2012). VM has been observed in several highly aggressive tumor types, including invasive breast cancer (Basu et al., 2006; Liu et al., 2013; Lee et al., 2014), hepatocellular carcinoma (Ma et al., 2011) and gallbladder carcinomas (Lu et al., 2013) etc... Importantly, the degree of VM correlates with poor clinical prognosis in many malignancies (Hendrix et al., 2003). Breast cancer is one of the most vascularized tumors and angiogenesis inhibitors may have theoretically produced satisfied therapeutic effects. However, the benefits are at best transitory, followed by malignant growth and progression of tumors. Accumulated evidence indicated that the development of VM is possibly a major obstacle for resistance to anti-angiogenesis therapy (Bergers et al., 2008). Hence, we address vasculogenic mimicry may be one of the tumor resistance mechanisms to anti-angiogenic therapy (van der Schaft et al., 2004; Bergers et al., 2008; Takano, 2012; Xu et al., 2012; Soda et al., 2013). Hence, 
it should be considered to develop new therapeutic agents that targeting VM for TNBC.

Although invasive breast cancer cells were found to display VM when cultured on matrigel, the mechanisms are not fully understood. Recently, experimental evidences have shown the importance of several key molecules which regulated VM information of aggressive malignant tumor cells. The epithelial-to-mesenchymal transition (EMT), as defined by loss of epithelial characteristics (E-cadherin) and gain of a mesenchymal phenotype (VE-cadherin), is a key step in the tumor metastasis process. As a"EMT trigger", Twist1 plays an essential role in metastasis. Recent works reported that Twist1 contributes to VM channels through promoting an epithelial-mesenchymal transition (EMT) in highly aggressive human tumor types (Yang et al., 2004; Sun et al., 2010; Ma et al., 2011; Zhao et al., 2011). Given that only tumor angiogenesis inhibitors have no effect on VM, which may indirectly elicit the emergence of distant metastasis ( $\mathrm{Xu}$ et al., 2012). Therefore, it's urgent to seek a novel therapeutic drug targeting VM to obtain greater clinical benefits of anti-angiogenesis therapies.

The grape seed proanthocyanidins (GSPs), a kind of promising botanical agents, have been reported to possess powerful anti-carcinogenic and/or anti-angiogenesis effects in different tumor models, which exhibit no apparent toxicity and genotoxic potential (Mantena et al., 2006; Sun et al., 2011a; Huang et al., 2012; Feng et al., 2014). Although GSPs has anti-carcinogenic effects in highly aggressive breast carcinoma cells, its effect on vasculogenic mimicry (VM) have not been explored. Therefore, in the present study, we investigated whether GSPs inhibit the development of vasculogenic mimicry (VM) and the regulating molecules in TNBC.

\section{Materials and Methods}

\section{Cell culture}

The human TNBC cells HCC1937 were acquired from the American Type Culture Collection (ATCC, Manassas, VA, USA). MCF-7 cells were obtain from frozen stocks routinely used and published previously (Yue et al., 2012), which characterized as ER-positive/PgR-positive luminal mammary carcinoma. Cells were cultured as monolayers in Roswell Park Memorial Institute (RPMI 1640, Gibco, USA) containing 10\% fetal bovine serum (Gibco BRL, Grand Island, NY), 100 $\mu \mathrm{g} / \mathrm{ml}$ penicillin and $100 \mu \mathrm{g} / \mathrm{ml}$ streptomycin (Invitrogen). Cells were incubated and maintained in a chamber with $95 \%$ air and $5 \% \mathrm{CO}_{2}$ at $37^{\circ} \mathrm{C}$. All experiments were performed on cells exponential phases.

\section{GSPs, composition, dissolution}

The grape seed proanthocyanidins (GSPs) are a natural extract of grape seeds, containing approximately $89 \%$ proanthocyanidins, with dimmers $(6.6 \%)$, trimers $(5.0 \%)$, tetramers $(2.9 \%)$ and oligomers $(74.8 \%)$, as described previously (Mantena et al., 2006; Nandakumar et al., 2008; Meeran et al., 2009). This extract is stable when refrigerated at $4^{\circ} \mathrm{C}$, with a shelf life of up to 2 years. GSPs were freshly dissolved in a spot of dimethylsulfoxide
(DMSO), then the compounds were added to the complete cell culture medium. The preparation of GSPs was required to achieve aims: the maximum concentration of solvent in media, $0.1 \%(\mathrm{v} / \mathrm{v})$ and treatment of cells with GSPs at various concentrations of $0.50 \mu \mathrm{g} / \mathrm{ml}, 100 \mu \mathrm{g} / \mathrm{ml}$, $200 \mu \mathrm{g} / \mathrm{ml}$.

\section{Cell proliferation Assay}

The effect of GSPs on the proliferation of HCC1937 cells was examined by the CCK8 assay, as previously described (Yue et al., 2012). Cultured HCC1937 cell suspensions were prepared prior to below experiment. In addition, we employed a Coulter counter to quantified cell numbers. Briefly, cells were seeded in a 96-well plate at a density of $1 \times 10^{4}$ cells per well in $100 \mu$ complete medium and incubated overnight. Then, GSPs were added into each well in order to achieve the final concentration ranging from 50 to $200 \mu \mathrm{g} / \mathrm{ml}$. After that, the cells incubated in a humidified atmosphere of $5 \% \mathrm{CO}_{2}$ at $37^{\circ} \mathrm{Cfor}$ a further $24 \mathrm{~h}$, $48 \mathrm{~h}$.At the end of the indicated time, cells were treated with CCK8 (10 $\mu \mathrm{l} /$ well, Sigma, USA) for an additional $2 \mathrm{~h}$, Finally, we recorded the absorbance at $450 \mathrm{~nm}$ using a microplate absorbance reader (Tecan, Safire II, Switzerland).

\section{Network formation assay in vitro}

Tubular network formation was measured by the three-dimensional Matrigel (BD Biosciences) culture as described previously (Zhang et al., 2014). Briefly, Matrigel (50 $\mu \mathrm{l} /$ well, BD Biosciences, USA) were added to 96-well culture plates and allowed to polymerize at $37^{\circ} \mathrm{C}$ for $1 \mathrm{~h}$, before the GSPs-treated cells were plated. Then, $100 \mu$ l of HCC1937 cells suspensions $\left(1.0 \times 10^{5} / \mathrm{ml}\right)$ were seeded onto the Matrigel coated plates for indicated time until forming an patterned networks in the control group. The representative images were captured using an Olympus IX50 microscope fitted with a digital camera (Nikon-Diafot)

\section{Western blotting in vivo}

We performed western blot analysis for Twist1, E-cadherin, VE-cadherin proteins from the tumor cells to explore the effect and molecular mechanism of GSPs on vasculogenic mimicry (VM), as previously reported (Mantena et al., 2006). After treatment of breast cancer cells for $24 \mathrm{~h}$ with or without GSPs, the cells were harvested, washed with cold PBS and lysed with ice-cold lysis buffer containing protease inhibitors. Equal amounts of proteins extracts were separated on $8 \%$ polyacrylamide gel and subsequently transferred onto polyvinylidene difluoride membrane (PVDF), then blocked with $5 \%$ non-fat milk in TBST. After being washed with TBS for three times, the membrane were incubated with different primary antibody against Twist 1 , E-cadherin and VE-cadherin (Boster, Wuhan, China, 1: 100 ) overnight at $4^{\circ} \mathrm{C}$, followed by incubation with a horseradish peroxidase-conjugated secondary antibody (浓度) at room temperature for one hour. The targeted protein bands were visualized on X-ray film using an enhanced chemiluminescence reagent system. $\beta$-action was monitored as a loading control. 
Effect of Grape Seed Proanthocyanidins on Tumor Vasculogenic Mimicry in Human Triple-negative Breast Cancer Cells

Statistical analysis

All data were expressed as the mean \pm standard deviation (SD). Statistical software SPSS 22.0 was used in the analysis. Statistical differences between groups were assessed using the one-way ANOVA. A value of $p<0.05$ was considered as statistically significant.

\section{Results}

Effect of GSPs on proliferation of HCC1937 cells

To assess whether GSPs have the ability to inhibit the proliferation of HCC1937 cells, the CCK8 assay was performed in vitro. As shown in Figure 1, incubation of HCC1937 cells with various concentration GSPs for up to $48 \mathrm{~h}$ resulted in a significant reduction in cells

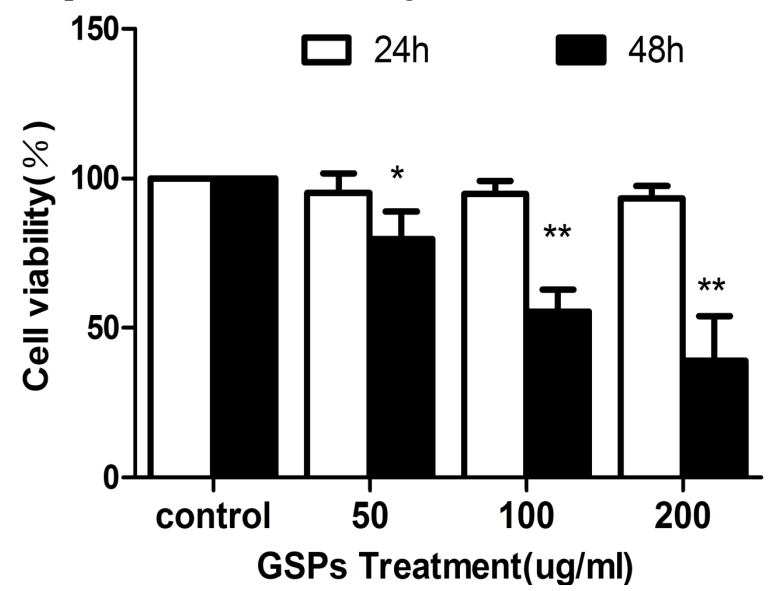

Figure 1. Treatment of HCC1937 Cells with Various GSPs Inhibits Cell Viability in a Dose-Dependent Manner. The cell viability was determined using the CCK8 assay. The results are presented as the mean \pm SD. mean of 6 replicates. Each cell viability experiment was repeated 3 times.The asterisk indicates a significant difference compared to the control (ANOVA, ${ }^{*} p<0.01,{ }^{*} p<0.001$ )

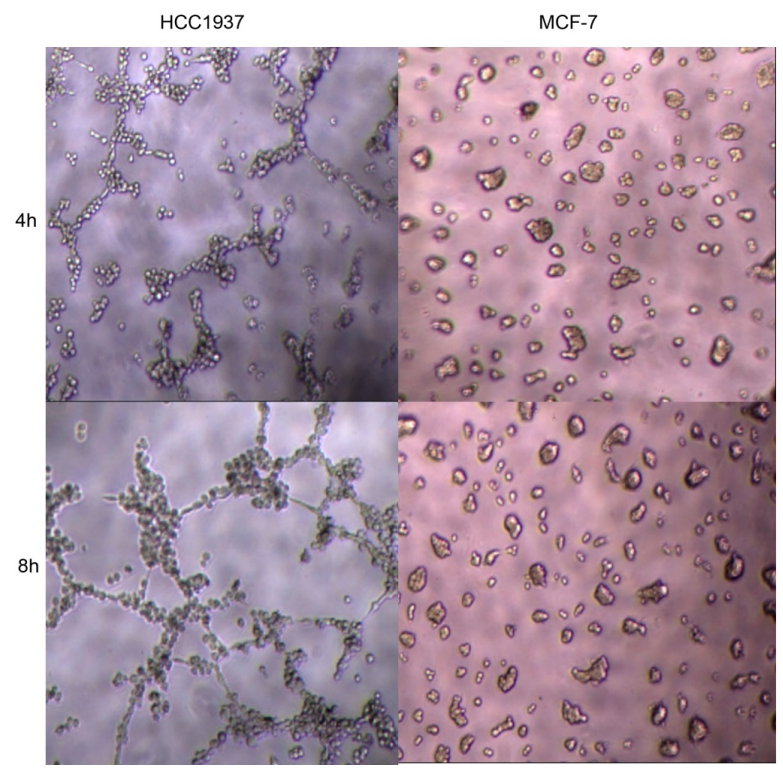

Figure 2. The Patterned Networks were Observed in Highly Invasive HCC1937 Cells Cultured in the 3D Matrix, which Evolved Dynamically and Anastomosed over a 4 to 8 hour Period ; There was not Notable for Poorly Aggressive MCF-7 Cells to form these Networks proliferation in a dose-dependent manner compared to non-GSPs-treated control cells, which were assigned a value of $100 \%$ viability. The effect of GSPs resulted in a 11.7-74.2\%reduction after $48 \mathrm{~h}(p<0.05-0.001)$. However, treatment of HCC 1937 cells with GSPs $(50-200 \mu \mathrm{g} / \mathrm{ml})$ for $24 \mathrm{~h}$ did not significantly change the proliferation ability under the same experimental conditions. Therefore, all further experiments were administrated not exceeding $24 \mathrm{~h}$.

GSPs inhibits the tube information of HCC1937 cells in vitro

Relative highly invasive HCC1937 cells generated the tubular-like structures when plated on Matrigel, which evolved dynamically over a 4 to 8 hour period (Figure 2). In contrast, patterned networks were absent for poorly aggressive MCF-7 cells under the same conditions (Figure 2). Treatment of HCC1937 cells with a lower dose $(50 \mathrm{ug} / \mathrm{ml})$ of GSPs had no significant effect on vascular channels at the indicated time (data not shown). We found that incubation with GSPs at the concentration of 100 and $200 \mathrm{ug} / \mathrm{ml}$ were reduce significantly the number of vascular channels compared to the cells which were not treated with GSPs (all $p<0.001$, Figure 3). These results suggested that GSPs inhibits vascular channel formation in breast cancer HCC1937 cells.

The influence of GSPs on the protein expression of Twist1, epithelial and mesenchymal biomarker in HCC1937

Twist1 has been identified as an important regulator of EMT, which in turn has been involved in the information of VM, cancer cell invasion and metastasis.To check whether GSPs affect Twist1 or EMT in HCC1937 cells, HCC1937 cells were incubated with or without GSPs for $24 \mathrm{~h}$. Thereafter, cell lysates were prepared for the western
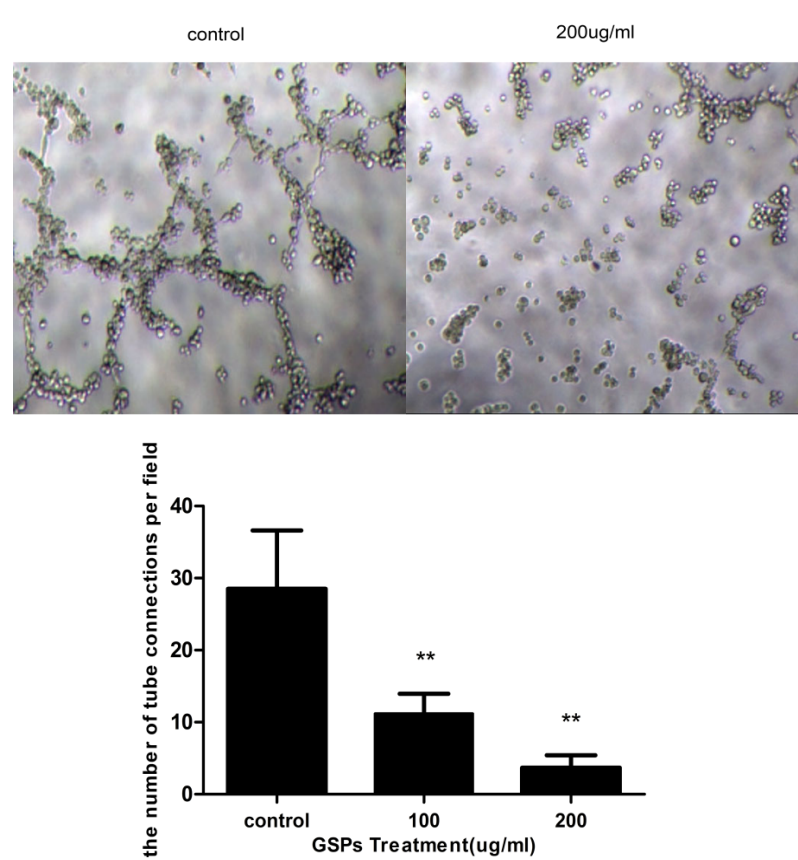

Figure 3. Effect of GSPs on the VM Information of HCC1937 Cell (24h, 400x). The vascular channels were quantified by counting the average number of tube connections in five randomly selected fields. Results were expressed as the mean \pm SD. of 6 wells from three independent experiments. $* * p<0.001$ vs vehicle control 

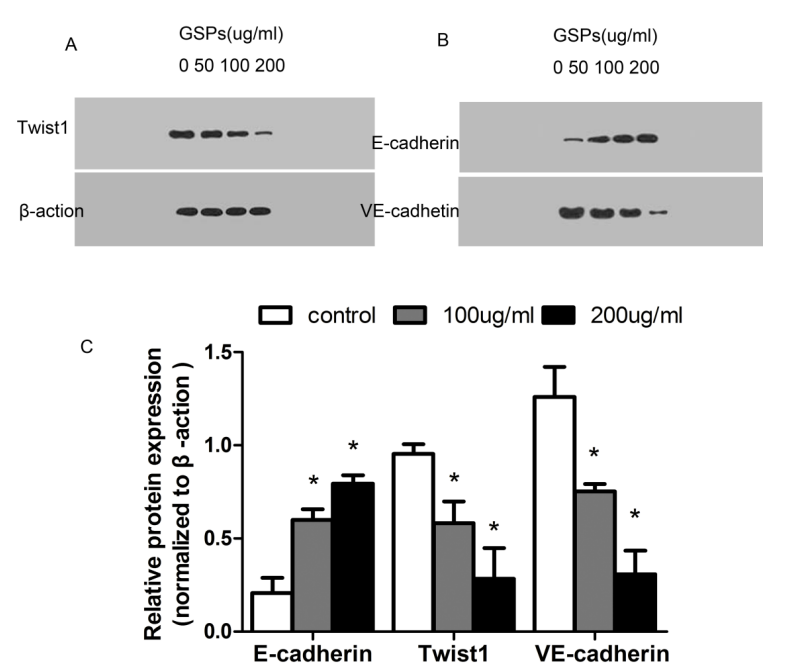

Figure 4. Effect of GSPs on VM-related Protein Expression in Breast Cancer Cells HCC1937. A) After treatment of cells with or without GSPs for 24h,the levels of Twist 1 protein were examined in cell lysates using western blot analysis. B) Treatment of HCC1937 cells with GSPs for 24h enhanced the levels of epithelial biomarkers (E-cadherin) while loss of mesenchymal biomarkers (VE-cadherin), in a dosedependent manner. C)The relative protein levels were expression after normalized by $\beta$-action. ${ }^{*} p<0.01$ vs control

blot analyses of VM-related protein. As shown in Figure 4, representative images are shown from three independent experiments. Our western blot analyses verified that the expression of Twist1 protein was significantly decreased $(p<0.01)$ in HCC1937 breast cells when treated with GSPs (100 and 200ug/ml). The level of mesenchymal biomarker, such as VE-cadherin, was significantly reduced after treatment with GSPs $(100$ and $200 \mathrm{ug} / \mathrm{ml})$ in a dosedependent manner $(p<0.01)$. In contrast, 100 and $200 \mathrm{ug} / \mathrm{ml}$ concentrations of GSPs could significantly enhanced the level of E-cadherin (an epithelial biomarker)compared to untreated control cells $(p<0.01)$. There was no significantly effect on VM-related protein (Twist1, VE-cadherin and E-cadherin)after treatment with GSPs (data not shown). In conclusion, these results indicated that GSPs inhibited the information of VM by downregulating Twist1, consequently triggering transition of the mesenchymal state to the epithelial state.

\section{Discussion}

As a heterogeneous subtype, triple-negative breast cancers (TNBCs) were defined by the lack of estrogen receptor and progesterone receptor, and non-amplification of Her/neu, accounting for approximately $10-15 \%$ of diagnosed breast cancer cases. Therefore, TNBCs lack effective targeted therapy agents and therapy is limited to chemotherapy. The poor clinical prognoses of TNBC appear to be related to an aggressive metastatic character and rapid recurrence after treatment. (Taylor et al., 2013; Han et al., 2014; Yoshida et al., 2014) Given this situation, the development of new treatment strategies for TNBC is need. As everyone knows, tumor growth and metastasis are thought to be angiogenesis-related processes. Traditionally, agents targeting the vascular endothelial growth factor (VEGF) may have satisfied therapeutic effects. Unfortunately, the benefits are at best transitory and may facilitate malignant growth and metastasis of tumors. (Soda et al., 2013; Zhang et al., 2014) Expect for traditional recognized tumor channels (angiogenesis, vasculogenesis), there exists another tubular network in highly aggressive tumors, which termed as vasculogenic mimicry (VM). The newly vascular channels are transdifferentiated from highly malignant tumor cells without the participation of endothelial cells. These channels may serve as an alternative means of tumor microcirculation. Accumulated evidence from many groups indicated that the tumor resistance to anti-angiogenic therapy may be explained in part by the development of VM hypothesis (Takano, 2012; Soda et al., 2013). Thus, it makes sense to develop a novel and accurate anti-vascular therapeutic agent targeting VM.

Proanthocyanidins are abundantly available in various parts of the plants, such as fruits, berries, bark and seeds. The seeds of the grape are particularly rich source of proanthocyanidins. In recent years, the grape seed proanthocyanidins (GSPs) has been hailed as a natural anti-carcinogenic agents in different tumor models, which indicate a low toxicity and have no genotoxic potential (Mantena et al., 2006; Nandakumar et al., 2008; Meeran et al., 2009; Huang et al., 2012). In this study, we further investigated the anti-VM activity of GSPs as a VM inhibitor for human TNBC cell HCC1937.The results have shown that highly aggressive TNBC cells HCC1937 were able to form vasculogenic-like network structures when cultured on a three-dimensional matrix; that poorly aggressive MCF-7 were unable to form the patterned networks with the same conditions, which were concordant with previous research (Basu et al., 2006). What is more, GSPs inhibited significantly proliferation of HCC 1937 cells and suppressed tubular-like structures in vitro. Therefore, we concluded that GSPs may be a potential anti-VM agent for human TNBC.

Although VM has been shown in a triple-negative breast cancer cells, molecular events underlying VM remain somewhat unclear. Therefore, understanding of mechanisms underlying VM would provide potential targets for new therapies of TNBC. Previous studies have revealed that several molecules or signaling pathways related to VM by aggressive malignant tumor, including PI3K, VEGF, EphA2, MMPs, Ln-5 $\gamma 2$, etc (Chen et al., 2012; Seftor et al., 2012; Lu et al., 2013). Recently, a considerable emphasis has been focused on the role of Epithelial-mesenchymal transition (EMT) in regulating VM information of aggressive malignant tumor cells. A hallmark of EMT is loss of epithelial markers such as E-cadherin and activation of mesenchymal markers such as VE-cadherin, Which promote tumor invasion, metastasis and recently found VM (Sun et al., 2010; Sun et al., 2011a). In the present study, GSPs treatment of HCC1937 cells showed a significantly transition of mesenchymal state to epithelial state. Taken together, these data suggested that the loss of E-cadherin and restoration of VE-cadherin can be an important reason for the anti-VM property of GSPs. As a EMT regulator, Twist1 overexpression correlated to the development of 
Effect of Grape Seed Proanthocyanidins on Tumor Vasculogenic Mimicry in Human Triple-negative Breast Cancer Cells

VM in highly aggressive human tumor types (Yang et al., 2004; Ma et al., 2011; Sun et al., 2011b). We assessed the expression of Twist 1 using western blot analysis. Our data demonstrate that the expression of Twist1 was significantly inhibited under the influence of GSPs. Collectively, GSPs, as a promising botanicals, may inhibit the expression Twist 1 , reducing breast cancer cells plasticity to VM cells by the epithelial-to-mesenchymal transition (EMT), thus inhibiting tumor growth and VM channels of TNBC.

\section{Acknowledgements}

We gratefully acknowledge Dr Zhong for the donation of GSPs. This study was supported by Department of Oncology, Affiliated Hospital of Qingdao University.

\section{References}

Basu GD, Liang WS, Stephan DA, et al (2006). A novel role for cyclooxygenase- 2 in regulating vascular channel formation by human breast cancer cells. Breast Cancer Res, 8, 69 .

Bergers G, Hanahan D (2008). Modes of resistance to antiangiogenic therapy. Nat Rev Cancer, 8, 592-603.

Chen Y, Jing Z, Luo C, et al (2012). Vasculogenic mimicrypotential target for glioblastoma therapy: an in vitro and in vivo study. Med Oncol, 29, 324-31.

Di Cosimo S, Baselga J (2010). Management of breast cancer with targeted agents: importance of heterogeneity. [corrected]. Nat Rev Clin Oncol, 7, 139-47.

Feng L-L, Liu B-X, Zhong J-Y, et al (2014). Effect of grape procyanidins on tumor angiogenesis in liver cancer xenograft models. Asian Pac J Cancer Prev, 15, 737-41.

Han J, Bae SY, Oh SJ, et al (2014). Zerumbone suppresses IL-1beta-induced cell migration and invasion by inhibiting IL-8 and MMP-3 expression in human triple-negative breast cancer cells. Phytother Res.

Hendrix MJ, Seftor EA, Hess AR, et al (2003). Vasculogenic mimicry and tumour-cell plasticity: lessons from melanoma. Nat Rev Cancer, 3, 411-21.

Huang S, Yang N, Liu Y, et al (2012). Grape seed proanthocyanidins inhibit colon cancer-induced angiogenesis through suppressing the expression of VEGF and Ang1. Int J Mol Med, 30, 1410-6.

Lee CH, Wu YT, Hsieh HC, et al (2014). Epidermal growth factor/heat shock protein 27 pathway regulates vasculogenic mimicry activity of breast cancer stem/progenitor cells. Biochimie, 104, 117-26.

Liu TJ, Sun BC, Zhao XL, et al (2013). CD133+ cells with cancer stem cell characteristics associates with vasculogenic mimicry in triple-negative breast cancer. Oncogene, 32, 544-53.

Lu XS, Sun W, Ge CY, et al (2013). Contribution of the PI3K/ MMPs/Ln-5gamma2 and EphA2/FAK/Paxillin signaling pathways to tumor growth and vasculogenic mimicry of gallbladder carcinomas. Int J Oncol, 42, 2103-15.

Ma JL, Han SX, Zhu Q, et al (2011). Role of Twist in vasculogenic mimicry formation in hypoxic hepatocellular carcinoma cells in vitro. Biochem Biophys Res Commun, 408, 686-91.

Maniotis AJ, Folberg R, Hess A, et al (1999). Vascular channel formation by human melanoma cells in vivo and in vitro: vasculogenic mimicry. Am J Pathol, 155, 739-52.

Mantena SK, Baliga MS, Katiyar SK (2006). Grape seed proanthocyanidins induce apoptosis and inhibit metastasis of highly metastatic breast carcinoma cells. Carcinogenesis, 27, 1682-91.
Meeran SM, Vaid M, Punathil T, et al (2009). Dietary grape seed proanthocyanidins inhibit 12-O-tetradecanoyl phorbol-13-acetate-caused skin tumor promotion in 7, 12-dimethylbenz[a]anthracene-initiated mouse skin, which is associated with the inhibition of inflammatory responses. Carcinogenesis, 30, 520-8.

Nandakumar V, Singh T, Katiyar SK (2008). Multi-targeted prevention and therapy of cancer by proanthocyanidins. Cancer Lett, 269, 378-87.

Redig AJ, McAllister SS (2013). Breast cancer as a systemic disease: a view of metastasis. J Intern Med, 274, 113-26.

Rhodes LV, Tate CR, Segar HC, et al (2014). Suppression of triple-negative breast cancer metastasis by pan-DAC inhibitor panobinostat via inhibition of ZEB family of EMT master regulators. Breast Cancer Res Treat, 145, 593-604.

Seftor RE, Hess AR, Seftor EA, et al (2012). Tumor cell vasculogenic mimicry: from controversy to therapeutic promise. Am J Pathol, 181, 1115-25.

Soda Y, Myskiw C, Rommel A, et al (2013). Mechanisms of neovascularization and resistance to anti-angiogenic therapies in glioblastoma multiforme. $J$ Mol Med, 91, 439-48.

Sun Q, Prasad R, Rosenthal E, et al (2011a). Grape seed proanthocyanidins inhibit the invasive potential of head and neck cutaneous squamous cell carcinoma cells by targeting EGFR expression and epithelial-to-mesenchymal transition. BMC Complement Altern Med, 11, 134.

Sun T, Sun BC, Zhao XL, et al (2011b). Promotion of tumor cell metastasis and vasculogenic mimicry by way of transcription coactivation by Bcl-2 and Twist1: a study of hepatocellular carcinoma. Hepatology, 54, 1690-706.

Sun T, Zhao N, Zhao XL, et al (2010). Expression and functional significance of Twist 1 in hepatocellular carcinoma: its role in vasculogenic mimicry. Hepatol, 51, 545-56.

Takano S (2012). Glioblastoma angiogenesis: VEGF resistance solutions and new strategies based on molecular mechanisms of tumor vessel formation. Brain Tumor Pathol, 29, 73-86.

Taylor MA, Davuluri G, Parvani JG, et al (2013). Upregulated WAVE3 expression is essential for TGF-beta-mediated EMT and metastasis of triple-negative breast cancer cells. Breast Cancer Res Treat, 142, 341-53.

van der Schaft DW, Seftor RE, Seftor EA, et al (2004). Effects of angiogenesis inhibitors on vascular network formation by human endothelial and melanoma cells. J Natl Cancer Inst, 96, 1473-7.

Xu Y, Li Q, Li XY, et al (2012). Short-term anti-vascular endothelial growth factor treatment elicits vasculogenic mimicry formation of tumors to accelerate metastasis. J Exp Clin Cancer Res, 31, 16.

Yang J, Mani SA, Donaher JL, et al (2004). Twist, a master regulator of morphogenesis, plays an essential role in tumor metastasis. Cell, 117, 927-39.

Yoshida T, Ozawa Y, Kimura T, et al (2014). Eribulin mesilate suppresses experimental metastasis of breast cancer cells by reversing phenotype from epithelial-mesenchymal transition (EMT) to mesenchymal-epithelial transition (MET) states. Br J Cancer, 110, 1497-505.

Yue L, Wang Y, Wang H, et al (2012). Inhibition of hepatocellular carcinoma cell growth by an anti-insulin-like growth factor-I receptor monoclonal antibody. Oncol Rep, 28, 1453-60.

Zhang JT, Sun W, Zhang WZ, et al (2014). Norcantharidin inhibits tumor growth and vasculogenic mimicry of human gallbladder carcinomas by suppression of the PI3-K/MMPs/ Ln-5gamma2 signaling pathway. BMC Cancer, 14, 193.

Zhao XL, Sun T, Che N, et al (2011). Promotion of hepatocellular carcinoma metastasis through matrix metalloproteinase activation by epithelial-mesenchymal transition regulator Twist1. J Cell Mol Med, 15, 691-700. 